\title{
SURFACE TENSION IN TEARS
}

\section{TENSIÓN SUPERFICIAL DE LA LÁGRIMA}

\author{
TIFFANY JM ${ }^{1}$
}

The preocular tear film has important roles as a nutritional route, as a smooth surface providing the first element of the refractive visual system, and as a medium for the protective and antimicrobial mechanisms of the ocular surface. Formation and stability of the film in both health and disease depend on the physical properties of the tears, including surface tension (ST).

The tear film entirely covers the exposed surface, with a bounding meniscus at all the surrounding eye margins. The film contains about $1 \mu \mathrm{l}$, the menisci about $2.9 \mu \mathrm{l}$, and a further volume lies under the eyelids. Although the exposed film area is large, ca. $1-3 \mathrm{~cm}^{2}$, it is very thin (range 2.7-11 $\mu \mathrm{m}$ (1) and on this scale gravitational forces promoting downward drainage are smaller than capillary forces, which are hence the dominant factor in holding the film onto the ocular surface. The film is formed and reformed at each blink, as part of a continuous cycle of (i) compression during the closing phase of the blink, where the upper and lower menisci merge and fresh fluid is added from under the upper lid, and (ii) opening, where a fresh film is drawn out of the menisci as the palpebral aperture widens. This opening and spreading phase has been modelled by Radke et al. (2), who derived the expression

$$
\mathrm{h}=2.123 \mathrm{R}(\mu \mathrm{U} / \sigma)^{2 / 3}
$$

where $\mathrm{h}=$ film thickness, $\mathrm{R}=$ meniscus radius of curvature, $\sigma=$ surface tension, $\mu=$ tear viscosity, $\mathrm{U}=$ lid velocity. Although its profile can change, the meniscus is always concave; the Young-Laplace equation indicates a pressure difference across this curved surface which tends to suck fluid back from the film into the meniscus. When the eye is fully open, this suction creates the «black line» of extreme thinness close to the meniscus, so that the preocular film is effectively isolated until the next blink. Punctal drainage is also inhibited between blinks.

Protein solutions have surface tensions lower than that of water or saline; this is true of the major tear proteins individually or in combination $(58.2$ $\mathrm{mN} / \mathrm{m}$ ) but the ST of tears is even lower (43-46 $\mathrm{mN} / \mathrm{m}$ ). Nagyová \& Tiffany (3) showed that extracting the lipids from tears raised ST to the proteinsonly value, but adding back the same lipids restored the tear ST value. Lipocalin is the only major tear protein with lipid-binding properties, and no free lipid is found in tears, so the lipocalin-lipid complex seems important, although it is still not known whether expression of ST involves lipid only (with the protein as a carrier to the surface), or the complex, or a «lipid-globule» structure for lipocalin in the surface phase. The specific nature of the lipids is also not fully known yet, but may include components not found in meibomian secretion.

Many methods of ST measurement require large volumes of tears, which are not readily available from dry-eye patients, and some earlier small-volume methods have been criticised for failure to ensure readings at equilibrium. The micro-technique of Tiffany et al. (4) is conveniently carried out in the collection capillary tube using as little as $0.2 \mu \mathrm{l}$ of tears. It should be remembered that here one is sampling the meniscus fluid, not the precorneal film, and that one is measuring the ST of the aqueous fluid only: if meibomian lipid is included in the collection the ST of the oil itself, and the oil/tears interfacial tension, will also be involved. Another widely-used method involves placing a droplet of tears on a defined surface (often contact lens materials) and measuring the equilibrium contact angle

\footnotetext{
${ }^{1} \mathrm{PhD}$. Nuffield Laboratory of Ophthalmology. University of Oxford, UK. E-mail: john.tiffany@eye.ox.ac.uk
} 
$\theta$ at the edge of the drop; Young's equation defines the balance of forces at the three-phase line as $\sigma_{\mathrm{a} / \mathrm{s}}$ $=\sigma_{1 / \mathrm{s}}+\sigma_{\mathrm{a} / 1} \cos \theta$, where $\sigma$ is ST and a, s, 1 refer to air, solid and liquid, allowing $\sigma_{\mathrm{a} / \mathrm{l}}$ to be determined. While this is satisfactory for laboratory investigation of artificial tears or drug vehicles, or of contact lens surfaces, only the micro-method (4) can currently give rapid results suitable for clinical purposes. The surfactant properties of tear components have also been investigated in the Langmuir trough by allowing proteins to diffuse from the bulk phase to the surface and interact with meibomian lipids, but the time-scale is so long that the relevance to rapid formation of the tear film after a blink is at present uncertain.

As shown in Tiffany et al. (4), the mean ST of tears from dry-eye patients is higher than ST of normals. In the ageing eye, levels of the major proteins are reported to decline, and this will raise ST to some extent even if lipocalin is unaffected. Tear film stability also declines with increasing age. This may argue for a direct connection between ST and stability, but adequate studies of the change in ST with age or dryness have yet to be made.

Many different formulations of artificial tears contain polymers, and some of these have ST com- parable to that of normal tears, while others are not very different from saline. At the time of writing, no peer-reviewed study seems to have been carried out to show that tear film stability is enhanced by lowering the surface tension of the tears or of an instilled artificial tear. Some newer types of artificial tears contain lipids, and if these include phospholipids ST will be altered; however, such solutions are formulated principally to try to reinforce the lipid layer of the tear film, rather than to produce a lower ST because this is known to improve tear film stability. More research is still needed to determine how best to tailor the surfactant properties to enhance stability.

\section{REFERENCES}

1. King-Smith PE, Fink BA, Hill RM, Koelling KW, Tiffany JM. The thickness of the tear film. Curr Eye Res 2004; 29: 357-368.

2. Creech JL, Do LT, Fatt I, Radke CJ. In vivo tear-film thickness determination and implications for tear-film stability. Curr Eye Res 1998; 17: 1058-1066.

3. Nagyova B, Tiffany JM. Components responsible for the surface tension of human tears. Curr Eye Res 1999; 19: 411.

4. Tiffany JM, Winter N, Bliss G. Tear film stability and tear surface tension. Curr Eye Res 1989; 8: 507-515. 